Barbara Bertisch-Möllenhoff
Daniel Meili
Milo Huber

\section{Nachweis von Hepatitis-C-Viruslast in gebrauchten Heroinspritzen}

\author{
Detection of Hepatitis C Viral Load in Used Syringes
}

\section{Zusammenfassung}

Ziel: Prüfung, ob in Spülflüssigkeit aus gebrauchten Heroinspritzen von Hepatitis-C-positiven Drogenkonsumenten HepatitisC-Viruslast nachweisbar ist. Diskussion der Hypothese, ob durch gemeinsames Zubereiten von Drogen in Gruppen bei Einsatz gebrauchter Spritzen die Weitergabe von Hepatitis-C-Viren über „indirect sharing“ möglich ist.

Methode: Gebrauchte Spritzen von sechs Hepatitis-C-infizierten Drogenkonsumenten wurden nach der Heroininjektion mit Kochsalzlösung durchspült; die Spüllösung wurde mittels PCRTechnik auf Nachweisbarkeit von Hepatitis-C-Viruslast untersucht. Die Höhe der Hepatitis-C-Viruslast im Blut der Patienten und in der Spülflüssigkeit wurde verglichen.

Teilnehmer: Die sechs Probanden sind Teilnehmer an einem Heroinverschreibungsprogramm in der Schweiz (Poliklinik Zokl2). Ergebnis: In allen Spülflüssigkeiten konnte eine beachtliche Hepatitis-C-Viruslast nachgewiesen werden (7530 bis 269825 cop/ $\mathrm{ml})$.

Schlussfolgerungen: Die gemessene Viruslast in gebrauchten Heroinspritzen ist ein weiteres Indiz dafür, dass das gemeinsame Zubereiten von Drogen mit Verwendung gebrauchter Spritzen ein Hepatitis-C-Infektionsrisiko darstellt.

\section{Schlliisselwörter}

Hepatitis C · Drogenkonsum · Heroin • Viruslast · PCR-Technik · Infektionsrisiko

\section{Abstract}

Aim: It is common for drugs to be prepared in groups and for used syringes to be involved in this process (indirect sharing). The aim was to detect viral load of rinsed syringes collected from hepatitis $\mathrm{C}$ positive heroin addicts. The following hypothesis is discussed: Is transmission of the hepatitis $C$ virus possible by indirect sharing?

Method: Used syringes from six hepatitis-C infected drug users were rinsed with $0.3 \mathrm{ml}$ of $\mathrm{NaCl}$ solution shortly after injection. This fluid underwent PCR testing for hepatitis $C$ viral load. The hepatitis $C$ viral load in the blood of the study participants was also measured.

Result: A significant hepatitis $C$ viral load was detected in all syringe samples. The range of viral loads was between 7,530 and $269,825 \mathrm{cop} / \mathrm{ml}$, while the blood samples yielded 1,503 to 23,067,000 cop/ml.

Conclusions: The detection of Hepatitis $\mathrm{C}$ viral load in fluid samples of used syringes is a further indication that preparation of drugs in groups with used syringes can be a risk for hepatitis $C$ transmission.

Key words

Hepatitis C · Drug Use · Heroin · Viral Load · PCR Testing · Transmission Risk

\section{Einleitung}

Bei Drogenabhängigen, die in Gruppen konsumieren, kommt es gehäuft zu Kooperation bei der Drogenzubereitung. Diese Kooperation kann die direkte Weitergabe von Nadeln und/oder
Spritzen beinhalten (direct sharing), aber auch Formen, bei welchen gemeinsames Auflösen und Teilen der Drogen praktiziert wird, ohne dass dabei gebrauchte Spritzen und Nadeln weitergegeben werden (indirect sharing). 
Trotz sehr ähnlicher Übertragungswege [1] kam es in den 90er Jahren, konträr zum Abfall der HIV-Inzidenz, zu einer epidemischen Ausbreitung der Hepatitis C [2-5]. In Abhängigkeit von dem Risikoverhalten wird bei intravenös Drogenkonsumierenden die Hepatitis-C-Durchseuchungsrate auf $60 \%$ bis $80 \%$ geschätzt [6]. Das „indirect sharing“ wird als möglicher und wichtiger Übertragungsgrund für Hepatitis C betrachtet [7].

Wie an anderer Stelle in diesem Heft von Meili et al. ausführlich geschildert [7], untersuchten wir an unserer Poliklinik für methadongestützte Behandlung, Zokl1, die genauen Konsumtechniken von 72 Teilnehmern am Methadonprogramm, die zum Zeitpunkt der Befragung intravenösen Beikonsum tätigten [8]. Dabei zeigte sich, dass bei der Drogenzubereitung in Gruppen Blutreste aus mehrfach verwendeten Spritzen verschiedene Utensilien wie Filter, Löffel und Spülwasser und dadurch die Drogenlösung kontaminieren können. Die Weitergabe von kleinen Blutmengen und darin allenfalls vorhandenen Viren ist möglich.

Zum Auflösen von Heroin, Kokain und Cocktails (Heroin-KokainGemisch) wird Wasser benötigt, welches meistens mittels Aufziehen in Spritzen abgemessen wird. Sollten dazu gebrauchte Spritzen verwendet werden, muss davon ausgegangen werden, dass ausgewaschene Blutreste und allenfalls vorhandene Viren in die Drogenlösung gelangen. Auch die Techniken des Aufziehens von Drogen (Frontloading, Backloading, Ausgleichen) ermöglichen eine Weitergabe von kleinen Blutmengen.

Wir fanden keine Hinweise in der Literatur, ob Hepatitis-C-Viruslast nachweisbar ist, wenn - analog zur Technik, die beim Zubereiten von Drogen praktiziert wird - eine kleine Restmenge Blut aus gebrauchten Spritzen in Wasser oder physiologischer Kochsalzlösung gelöst wird. Es liegen lediglich Untersuchungen über den Nachweis von Hepatitis-C-Viruslast und -Antikörpern in bis zu acht Monate alten Spritzen [9] sowie über den Nachweis von Hepatitis-C-Antikörpern in bis zu fünf Wochen alten Spritzen und Blutflecken [10] vor.

Ziel der Studie war es zu untersuchen, ob in der Spülflüssigkeit von gebrauchten Spritzen von HCV-Positiven eine messbare Hepatitis-C-Viruslast vorhanden ist.

\section{Material und Methode}

Bei sechs Hepatitis-C-positiven HIV-negativen Patienten, die sich in ärztlich kontrollierter, intravenöser Heroinsubstitutionsbehandlung in der Poliklinik Zokl2 befinden, bestimmten wir die Hepatitis-C-Viruslast im Blut. Die Hepatitis-C-Serokonversion war bereits von früher bekannt; keiner der Patienten befand sich in einer medikamentösen antiviralen Behandlung von Hepatitis C.

Wir nahmen eine von den Patienten unmittelbar zuvor nach gewohnter Technik zur intravenösen Injektion gebrauchte Spritze und entfernten größere Restmengen von Flüssigkeit, indem wir wenig Luft aufzogen und den Kolben durchdrückten. Anschließend zogen wir mit aufgesetzter Originalnadel 0,3 ml physiologische Kochsalzlösung auf. Diese Flüssigkeit wurde innerhalb einer halben Stunde in das immunologische Labor transportiert und sogleich untersucht.

Bei den ersten drei Patienten lagen zwischen der Bestimmung der Viruslast im Blut und in der Spülflüssigkeit zwei bis sieben Wochen, bei den letzten drei Patienten wurden diese Tests simultan durchgeführt.

Die Spülflüssigkeit zeigte in allen Fällen eine leichte bis stärkere rötliche Einfärbung.

\section{Resultate}

Das Ergebnis der Studie ist Tab.1 zu entnehmen.

Bei allen Probanden war im Blut eine Hepatitis-C-Viruslast nachweisbar. Ebenso konnte in allen Spülflüssigkeitsproben eine Hepatitis-C-Viruslast gemessen werden. Dabei zeigt sich bei allen Bestimmungen, außer bei Patient Nr. 3, eine deutlich geringere Viruslast in der Spülflüssigkeit im Vergleich zur Blutmessung. Bei Patient 3 ist das Ergebnis hingegen umgekehrt; allerdings lagen 18 Tage zwischen der Bestimmung der Viruslast im Blut und derjenigen in der Spülflüssigkeit.

\section{Diskussion}

Unsere Studie belegt, dass in der Spülflüssigkeit von gebrauchten Spritzen Hepatitis-C-positiver Drogenkonsumenten eine beachtliche Menge Hepatitis-C-Viren oder -Virusmaterial nachgewiesen werden kann. Bereits verwendete Spritzen finden beim gemeinsamen Zubereiten von Drogen in Gruppen zum intravenösen Konsum sehr oft Anwendung. Wie in unserem Experiment wird häufig Flüssigkeit in nicht oder nur mangelhaft gereinigte Spritzen aufgezogen, um damit die Drogen aufzulösen. Handelt es sich dabei um Spritzen von Hepatitis-C-Infizierten, können auf diesem Weg neben der Drogenlösung alle beteiligten Utensilien wie Filter, Löffel und Rührwerkzeug mit Viren kontaminiert werden. Bei der Technik des Aufteilens der Drogenlösung

\begin{tabular}{|c|c|c|c|c|}
\hline 2 & 23. Mai 00 & 228410 & 6. Juli 00 & 17519 \\
\hline 3 & 27. Jul. 00 & 1503 & 14. Aug. 00 & 7530 \\
\hline 5 & 4. Dez. 00 & 8819050 & 4. Dez. 00 & 67337 \\
\hline 6 & 7. Dez. 00 & 23067000 & 7. Dez. 00 & 69667 \\
\hline
\end{tabular}

Tab. 1 Hepatitis-CViruslast in Blut und in Spülflüssigkeit aus gebrauchten Spritzen 
können nochmals Blutpartikel direkt aus gebrauchten Spritzen unter den Beteiligten weitergegeben werden. Die detaillierten Praktiken sind von Meili et al. in einem anderen Artikel in diesem Heft aufgezeigt [7].

Einschränkend muss angefügt werden, dass keine sichere Methode existiert, um die Replikationsfähigkeit dieser Viren bzw. dieses Virusmaterials zu bestimmen. Die PCR-Messung erfasst Oberflächenbestandteile sowohl aktiver wie auch inaktivierter Viren. Bei jeder Infektion braucht es eine Mindestanzahl Keime, die für eine Übertragung der Erkrankung nötig ist. Für Hepatitis C liegen beim Menschen dazu keine Angaben vor.

Wir rekrutierten Drogenabhängige mit einer positiven Viruslast im Blut, gemessen einige Wochen vor oder simultan zur Messung der Spülflüssigkeit. Erwartungsgemäß war in der Spülflüssigkeit die Virenzahl in fünf Proben wesentlich tiefer als im Blut. Bei einem Probanden war die Viruskonzentration in der Spülflüssigkeit jedoch höher. Dies dürfte durch die geringe Zahl von Viren in der Blutmessung (relativ großer Messfehlerbereich) und/oder durch den zeitlichen Abstand der Bestimmungen mit der dadurch möglichen Schwankung verursacht sein.

Selbst wenn die Viren durch das Erwärmen der Drogenlösung inaktiviert würden, bliebe sehr oft ein Risiko der Virus-Transmission bestehen, da der Lösung häufig erneut kaltes Wasser, wiederum aus Spritzen, beigemengt wird. Auch beim anschließenden Teilen kann die flüssige Droge wieder mit fremden Spritzen und Nadeln in Kontakt kommen und zusätzlich kontaminiert werden (Frontloading, Backloading, Ausgleichen über Löffel).

\section{Schlussfolgerung}

Aufgrund international publizierter epidemiologischer Untersuchungen [7] und der Nachweisbarkeit von Hepatitis-C-Viruslast in gebrauchten Spritzen muss das „indirect sharing“ mit mehrfach verwendeten Utensilien als erhebliches HepatitisC-Risiko eingestuft werden. Dennoch finden diese Erkenntnisse in den Präventionskampagnen wenig Augenmerk. Die Präventionsbotschaft lässt sich nicht so stark verkürzen wie bei HIV mit dem Slogan: „Kein Spritzen- oder Nadeltausch.“

Die Botschaft: „Bei jeder Injektion, ob in Gruppen oder alleine, eine neue Spritze, neue Nadel, neue Filter, NaCl-Wasser aus Einmalgebinde und saubere Löffel“ bedarf bei den Anwendern einer detaillierten Erklärung. Dazu müssen Drogenfachleute entsprechend informiert sein. Die Gewissheit, dass in der Spülflüssigkeit von gebrauchten Spritzen von Hepatitis-C-Infizierten HepatitisC-Virusmaterial nachgewiesen werden kann, ist dienlich zur Veranschaulichung des Infektionsrisikos beim ,indirect sharing“.

\section{Danksagung}

Wir bedanken uns bei Frau Dr. H. Joller-Jemelka, Abteilung für Klinische Immunologie, Medizinische Klinik der Universität Zürich, bei Herrn Dr. Thomas Steffen, Facharzt für Prävention und Gesundheitswesen, Basel, und bei Herrn Dr. Jen Wang, Institut für Sozial- und Präventivmedizin, Universität Zürich, für die fachliche Unterstützung.

\section{Literatur}

${ }^{1}$ Grob PJ, Negro F, Renner EL (im Namen der SEVHEP). Hepatitis CVirus-Infektion. Praxis 2000; 89: 1587-1604

2 Steffen T, Gutzwiller F. Hepatitis B und C bei intravenös Drogenkonsumierenden in der Schweiz. Praxis 1999; 88: 1937-1944

${ }^{3}$ Infektionskrankheiten in der Schweiz 1999. BAG 2001; 2: $27-28$

4 Überwachung der Infektionskrankheiten: Akute Hepatitiden in der Schweiz 1994-1995. Bundesamt für Gesundheit (BAG). Bulletin 1997; 16 (28.4.1997)

${ }^{5}$ Somaini B, Wang J, Perozo M, Kuhn F, Meili D, Grob P, Flepp M. A continuing concern: HIV and hepatitis testing and prevalence among drug users in substitution programmes in Zurich, Switzerland. AIDS care 2000; 12 (4): $449-460$

${ }^{6}$ Huber M, Schmid P, Vernazza P, Meili D. Gründe für die ausbleibende Hepatitis C Behandlung bei Drogenabhängigen in Opiatsubstitution. Suchttherapie Supplement 2002; S27-S30

${ }^{7}$ Meili D, Marcinko J, Bertisch-Möllenhoff B, Huber M, Davatz F, Wang J. Mögliche Wege der Übertragung viraler Erkrankungen beim gemeinsamen Zubereiten von Drogen zum i.v.-Konsum - Konsequenzen für die Prävention. Suchttherapie Supplement 2002; S20-S26

${ }^{8}$ Marcinko J. Intravenöser Drogenkonsum: Genau betrachtete Praktiken des Injektionsverhaltens; eine detaillierte Befragung von Methadonbezügern. Inaugural-Dissertation, Institut für Sozial- und Präventivmedizin, Originalarbeit unter: www.arud-zh.ch. Zürich: Universität Zürich 1999

${ }^{9}$ Heimer R, Khoshnood K, Jariwala-Freeman B, Duncan B, Harima Y. Hepatitis in used syringes: The limits of sensitivity of techniques to detect hepatitis B virus (HBV) DNA, hepatitis C virus (HCV) RNA, and antibodies to HBV core and HCV antigens. J Infect Dis 1996; 173: $997-1000$

${ }^{10}$ Cattaneo C, Nuttall PA, Sokol RJ. Detection of HIV, Hepatitis B and Hepatitis $\mathrm{C}$ markers in discarded syringes and bloodstains. Science and justice 1996; 36 (4): $271-274$ 